\title{
Unified Analysis Model for Microstrip and Coupled lines Structures Utilizing Artificial Intelligence Techniques
}

\author{
H. Taher \\ Electronic engineering department \\ National university of Ireland, \\ Maynooth, Ireland \\ htaher@eeng.nuim.ie
}

\author{
R. Farrell \\ Electronic engineering department \\ National university of Ireland, \\ Maynooth, Ireland \\ $\underline{\text { rfarrell@eeng.nuim.ie }}$
}

\begin{abstract}
Planar structures such as single microstrip Line (SML) and microstrip coupled line (MCL) are basic components in RF circuits and systems. Traditionally, analysis parameters of SML and MCL, an effective permittivity $\left(\varepsilon_{e f f}\right)$ and characteristic impedance $\left(Z_{c}\right)$, are obtained using piecewise empirical functions. The intervals boundaries depend on the line width to the substrate high ratio (w/h) value. In this paper, Artificial Neural Network (ANN) is used to build unified, fast and accurate model for $\varepsilon_{e f f}$ and $Z_{c}$ of SML and MCL. The built ANN model is validated with the empirical models. Very accurate consolidated model for both of SML and MCL is obtained.
\end{abstract}

Keywords- Planar structures; Microstrip coupled lines; Artificial neural networks; microstrip lines

\section{INTRODUCTION}

A planar geometry implies that the characteristics of the element can be determined from the dimensions in a single plane. Several configurations for microstrip lines have been realized and some of these lines support transverse electromagnetic (TEM) modes, other hybrid or higher order modes. The circuits realized by any of these lines or combination of them have distinct advantages such as light weight, small size, improved performance, better reliability and reproducibility and low cost [1]

SML and MCL have become the best known and most widely used planar transmission line for RF and Microwave circuits. This popularity and widespread use are due to its planar nature, ease of fabrication using various processes, easy integration with solid-state devices, good heat sinking, and good mechanical support.

The study of TEM or quasi-TEM transmission MCL is important in microwave and digital electrical engineering. Traditionally, MCL have been extensively used in RF circuits to design directional couplers and all sort of filters. The application of coupled-line theory was not limited to microwave circuits; it had a wide impact on digital and telecommunications circuits too.

Knowing $\varepsilon_{e f f}$ and $Z_{c}$ of SML allows us to easily simulate or calculate circuit performance. Concerning the MCL, the even and the odd effective permittivity $\left(\varepsilon_{\text {effe }}, \varepsilon_{\text {effo }}\right)$ and the even and the odd characteristic impedance $\left(Z_{e}, \mathrm{Zo}\right)$ are the characteristics parameters that have to be extracted [1].

These parameters are usually calculated using ElectroMagnetic (EM) simulations. EM simulators utilize numerical techniques such as Finite difference (FD), Finite Elements (FE), Method of Lines (MOL) to obtain the characteristic parameters. Although accurate values are obtained, long simulation time is needed [2]-[6]. Therefore these techniques are not attractive for computer aided design (CAD). Using closed form equations is an alternative solution [7]-[10]. There is separate model for every component. Moreover, there are multi interval definitions per each model. It would be beneficial if there is one conjoined model for both components and does not fork for many definitions.

Artificial intelligent techniques are the candidates to develop fast, flexible and accurate models to be used in analyzing planer structures. Among these techniques, ANN is considered the most efficient techniques in building the required models. ANN is used as non linear function regression tool. ANN mimics the human brain in many sides:(i) The neurons or nodes are considered as processing elements (ii) each node is connected to other neurons with links (iii) The strengths of links are obtained through learning phase. Accurate and efficient microwave circuit components and microstrip antennas have been designed with the use of ANNs [11]-[13].

In this paper, ANN is used to build unified model for SML and MCL simultaneously. Excellent prediction for the analysis parameters is achieved using ANN model.

The paper is organized as follows; comprehensive study of the empirical model is presented in section two. In section three, basic theory of ANN is demonstrated. ANN model is validated in section four. Finally, the conclusions are drawn in section five.

\section{EMPRICAL MODELS OF SML AND MCL}

A SML, shown in Figure 1, is a printed circuit construction, consisting of a path of copper or other conductor on an insulating substrate. There is a "backplane" on the other side of the insulating substrate, formed from a similar conductor. It is used to transmit microwave signals. The microstrip line is the 
most commonly used microwave integrated circuit (MIC) transmission line. It has many advantages such as low cost, small size, ease of active device integration.

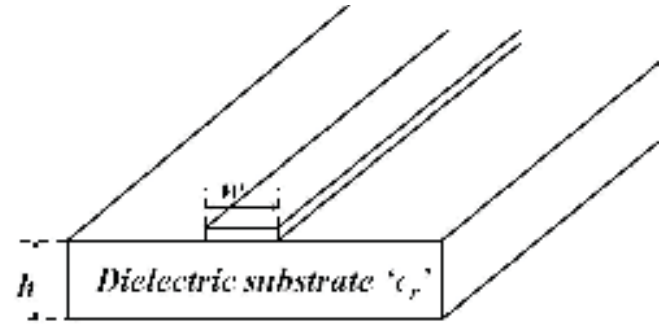

Fig. 1 SML structure.

$\varepsilon_{\text {eff }}$ of SML can be interpreted as the dielectric constant of homogenous medium that replaces the air and dielectric region of SML shown in Fig.1. The $\varepsilon_{\text {eff }}$ value is approximately given by [7],

$$
\varepsilon_{\text {eff }}=\frac{\varepsilon_{r}+1}{2}+\frac{\varepsilon_{r}-1}{2} \frac{1}{\sqrt{1+12 \frac{h}{w}}}
$$

where $\varepsilon_{r}$ is the substrate relative dielectric constant, ' $w$ ' is the width of the line and ' $h$ ' is the substrate height.

On the other hand $Z_{c}$ is obtained by the following equation,

$$
Z_{c}= \begin{cases}\frac{60}{\sqrt{\varepsilon_{\text {eff }}}} \ln \left(\frac{8 h}{w}+\frac{w}{4 h}\right) & \text { for } \frac{w}{h} \leq 1 \\ \frac{120 \pi}{\sqrt{\varepsilon_{\text {eff }}\left[\frac{w}{h}+1.393+0.667 \ln \left(\frac{w}{h}+1.444\right)\right]}} & \text { for } \frac{w}{h} \geq 1\end{cases}
$$

On the other hand, MCL consist of two signal conductor on the top side of substrate, as shown in Figure 2. On the bottom of the substrate there is ground plane. There are two main application areas for coupled-line theory in electrical engineering.

The first is in microwave engineering, where the coupling between the line elements is enhanced in such way that coupled lines can be used to design directional couplers, electrical filters, and so forth. The second is in digital and telecommunications engineering, where the coupling between the lines is controlled so that the signals on each line propagate with minimum distortion on the pulse shape.

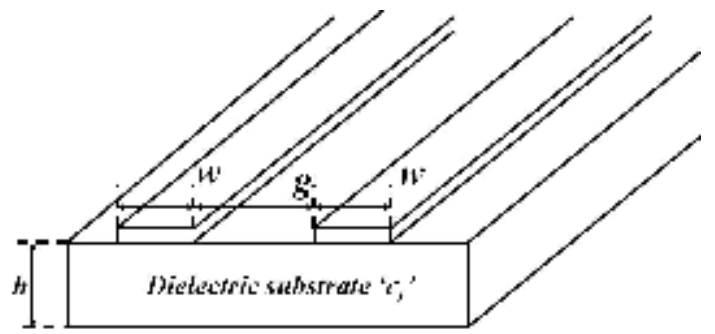

Fig. 2 MCL structure.

The $\varepsilon_{\text {effe }}, \varepsilon_{\text {effo }}$ and $Z_{e}$, Zo analysis equations are addressed in [10]. They are not listed here because they include more than 70 variables and constants. Each variable and constant has its own definition. Therefore, a much wider space is needed.

\section{ARTIFICIAL NEURAL NETWORK}

The ANN, shown in Figure 3, is constructed through learning from a set of input/output data (training set). After training, the ANN is able to generalize the relationship between the input and output, in the sense that for a given input value, which is not in the training set, it can predict the corresponding output.

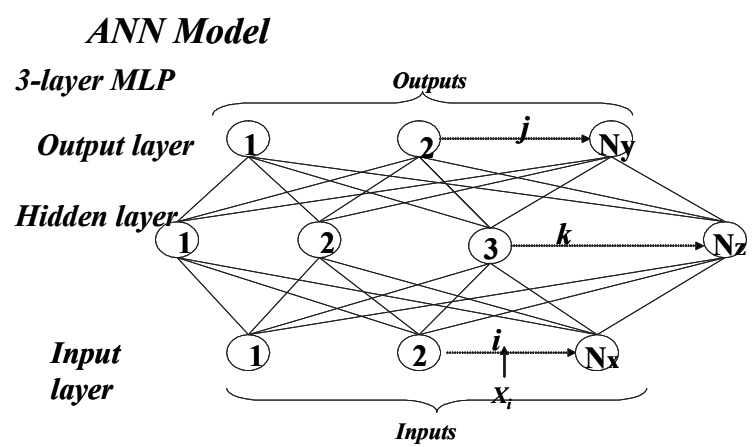

Fig. 3. Schematic for ANN.

The mapping between the input vector $x$ with $N_{x}$ the number of input neurons, and the output vector $y$ with $N_{y}$ the number of output neurons, can be determined as follows:

The inputs to the hidden layer are the $\gamma_{k}$, calculated from the input variables by:

$$
\gamma_{k}=\left(\sum_{i=1}^{N_{x}} x_{i} \omega_{k i}\right)+\theta_{k}, k=1,2, \ldots . ., N z
$$

Where $N_{z}$ is the number of neurons in the hidden layer, $\omega_{k i}$ is the weighting factor and $\theta_{k}$ is the bias term.

Let the activation function of the hidden layer be the sigmoid function, where

$$
f(\zeta)=\frac{1}{1+e^{-\zeta}}
$$

Then the output from the $k_{t h}$ neuron of the hidden layer,

$$
z_{k}=f\left(\gamma_{k}\right)
$$

the output of the $j_{t h}$ neuron in the output layer is,

$$
y_{j}=\left(\sum_{k=1}^{N_{z}} z_{k} \omega_{j k}\right)+\eta_{j}, \quad j=1,2, \ldots, N_{y}
$$

with $\omega_{j k}$ being the weighting factor and $\eta_{j}$ the bias term.

In fact, the training process is an optimization problem to find the best values for $\omega_{k i}, \theta_{k}, \gamma_{j k}$ and $\eta_{j}$ to minimize the objective function, which is the difference between the output from the ANN and the training data. The ANN is trained using 
the back-propagation algorithm [14], as implemented in the Neuro-Modeler program [15].

\section{ANN BASED MODEL FOR ANALYSIS PARAMETERS OF SML AND MCL}

The geometrical and physical properties of the structures, $\varepsilon_{r}, \frac{w}{h}$ and $\frac{g}{h}$, are the inputs of the ANN model. Since there is no role for the $\frac{g}{h}$ input in calculating the SML analysis parameters, therefore this input takes constant value in case of SML training. On the other hand, the analysis parameters, $\varepsilon_{e f f e}$, $\varepsilon_{\text {effo }}$ and $Z_{e}, Z_{o}$ are the outputs of ANN. The $\varepsilon_{\text {eff }}$ and $Z_{c}$ output of SML is incorporated into $\varepsilon_{e f f o}$ and $Z_{o}$ data. The schematic of the developed ANN is shown in Fig. 4.

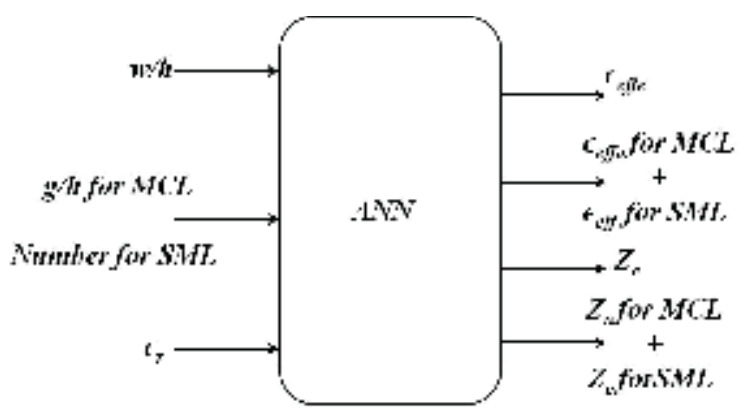

Fig. 4. Unified ANN model for SML and MCL.

To generate training and test data which are needed to build the ANN, the equations in [8] are used for SML case. The data sets are collected from $0.1 \leq w / h \leq 10.1$ and $1 \leq \epsilon_{r} \leq$ 10.5 range. Total 1912 data set are used in training phase and another different 360 data sets are utilized in the test stage. Concerning MCL case, the data are obtained using formulas in [10]. The data set are gathered from $0.1 \leq g / h \leq 10.1,0.1 \leq$ $w / h \leq 10$ and $1 \leq \epsilon_{r} \leq 10.5$ ranges. Total 2311 data sets are used in training phase and another different 900 data sets are utilized in the test phase.

3-layers ANN of the multilayer preceptron (MLP) kind is used. The input layer consists of the three neurons. On the other hand, there are only four neurons at the output layer. Regarding the hidden layer, it consists of 40 neurons. The number of hidden layer neurons is chosen such that minimum test error is obtained with minimum neurons to lower the computational cost.

To assess the built model, root mean square error (RMSE) for each model is calculated using the following formula;

$$
R M S E \%=\sqrt{\frac{1}{N_{t}} \times \sum_{i=1}^{N_{t}}\left(\frac{T_{d i}-O_{m i}}{T_{d i}}\right)^{2}} \%
$$

where, $N_{t}$ is number of test points, $T_{d i}$ is the value of the modeled parameter at test data point ' $i$ '. $O_{m i}$ is the output of the built model at the same point ' $i$ '. It is worth noting that the points used in calculating the RMSE are never utilized in the training phase. Table I lists the RMSE for every structure. The highest RMSE is noticed in case of $Z_{o}$, it amounts to $5.5 \%$.
More scoping on the behavior of the ANN technique in modeling SML and MCL is demonstrated using the comparisons between all the analysis parameters that resulted from ANN techniques and that used in the test phase. $\varepsilon_{e f f}$ and $Z_{c}$ of SML are shown in Figs. 5-6. Excellent agreement is noticed between the output parameters from the ANN model and the corresponding ones as calculated with the empirical models.

TABLE I. RMSE\% OF THE TRAINED ANN MODEL

\begin{tabular}{|c|c|c|}
\hline $\begin{array}{c}\text { Structure } \\
\text { Parameter }\end{array}$ & $\begin{array}{c}\text { MSL } \\
(\mathrm{RMSE} \%)\end{array}$ & $\begin{array}{c}\text { MCL } \\
(\mathrm{RMSE} \%)\end{array}$ \\
\hline$\varepsilon_{\text {effo }}$ & 0.9 & 1.2 \\
\hline$\varepsilon_{\text {effe }}$ & $\mathrm{NA}$ & 3.5 \\
\hline$Z_{o}$ & 4.9 & 5.5 \\
\hline$Z_{e}$ & $\mathrm{NA}$ & 4.5 \\
\hline
\end{tabular}

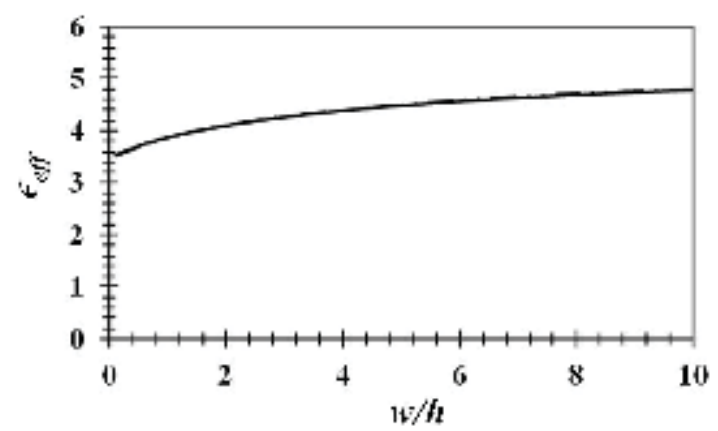

Fig. 5 Comparison between $\epsilon_{\text {eff }}$ of SML resulted from empirical model (-), ANN model (- -) $\epsilon_{r}=5.5$.

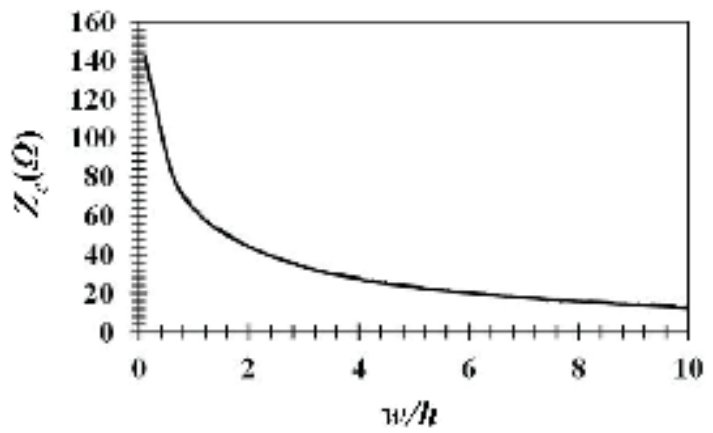

Fig.6 Comparison between $Z_{c}$ of SML resulted from empirical model (-), ANN model (- $) \epsilon_{r}=5.5$.

Regarding MCL analysis parameters, they are depicted in Figs. 7-10 for $\varepsilon_{\text {effo }}, \varepsilon_{\text {effe }}$ and $Z_{o}, Z_{\mathrm{e}}$ respectively. The analysis parameters are assessed against $\frac{g}{h}$. Excellent accuracy of the ANN based model in predicting the analysis parameters of MCL is observed. The behavior of the ANN model against $\frac{w}{h}$ has the same amount of accuracy. In general, the difference between the ANN output and the test data is attributed to inherent inevitable training fitting error. 


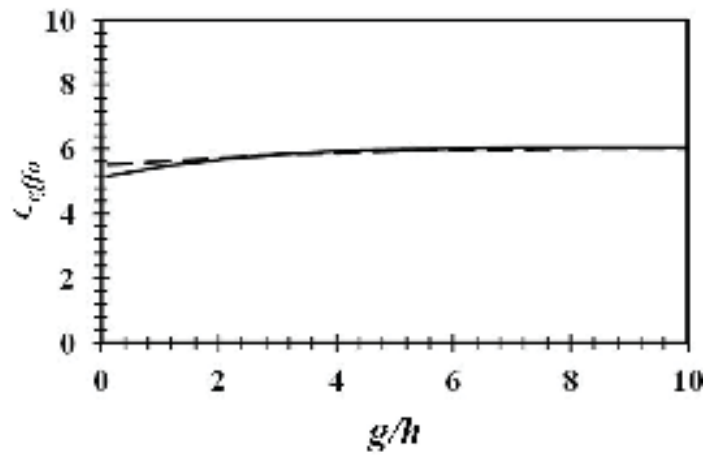

Fig. 7 Comparison between $\epsilon_{\text {effo }}$ of MCL resulted from empirical model (-), ANN model (- -) $\epsilon_{r}=10$ and $\frac{w}{h}=0.1$.

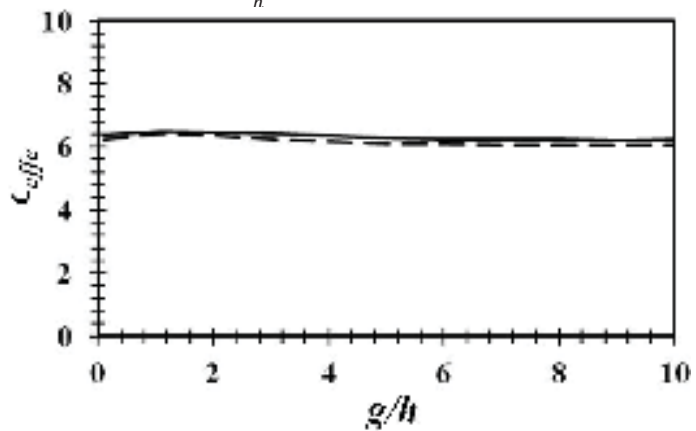

Fig. 8 Comparison between $\epsilon_{\text {effe }}$ of MCL resulted from empirical model (-), ANN model (- $) \epsilon_{r}=10$ and $\frac{w}{h}=0.1$.

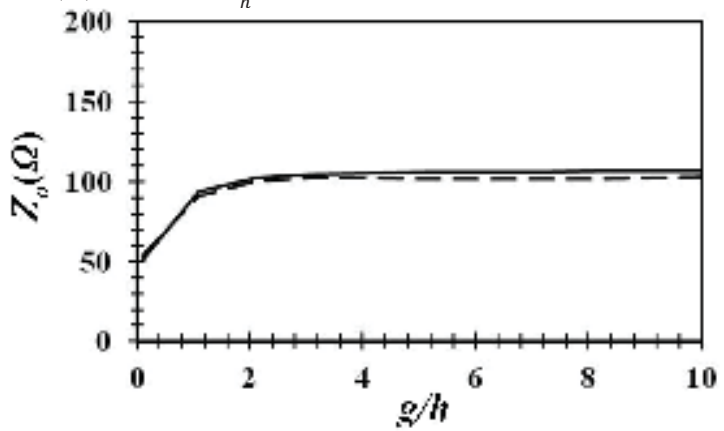

Fig. 9 Comparison between $Z_{o}$ of MCL resulted from empirical model (-), ANN model (- $) \epsilon_{r}=10$ and $\frac{w}{h}=0.1$.

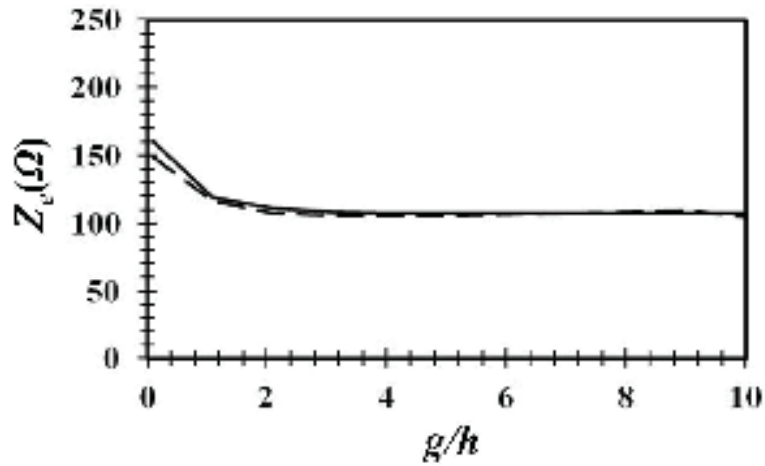

Fig. 10 Comparison between $Z_{e}$ of MCL resulted from empirical model (-), ANN model (- -) $\epsilon_{r}=10$ and $\frac{w}{h}=0.1$.

\section{CONCLUSION}

In this work, ANN technique is used to analyze SML and MCL simultaneously. Unified ANN model is developed to calculate all analysis parameters of both structures. Error study is performed to the obtained ANN model. This study reveals that the accuracy in predicting the analysis parameters is very high for all parameters. The maximum obtained test error is $5.5 \%$. It is possible to use training data extracted from electromagnetic simulators, and consequently, the built ANN model would have the high accuracy of full wave solutions methodologies. Based on that, ANN based model is an efficient tool to analyze SML and MCL simultaneously.

\section{ACKNOWLEDGMENT}

This research is supported by Science Foundation Ireland under Grant No. 10/CE/I1853. The authors appreciatively acknowledge this support. Dr. Hany is also Associate Prof. Researcher at Electronic Research Institute (ERI) -Giza, Egypt.

\section{REFERENCES}

[1] D. M. Pozar, Microwave Engineering, John Wiley \& Sons, Inc., New York, 2005.

[2] X. Zhang, J. Fang, K.K. Mei, and Y. Liu, "Calculation of the dispersive characteristics of microstrips by the timed omain finite-difference method," IEEE Trans MTT., vol. 36, pp. 263-267, 1988.

[3] M.S. Alam, Koshiba,M. Hirayama, K. and Hayashi,Y, "Hybrid-mode analysis of multilayered and multiconductor transmission lines," IEEE Trans MTT., vol. 45, pp. 205-211, 1997.

[4] G. Cano, F. Medina, and M. Horno, "Efficient spectral domain analysis of generalized multistrip lines in stratified media including thin, anisotropic, and lossy substrates," IEEE Trans MTT., vol. 40, pp. 217-227, 1992.

[5] C-I G. Hsu, R.F. Harrington, K.A. Michaliski, and D. Zheng, "Analysis of multiconductor transmission lines of arbitrary cross section in multilayered uniaxial media," IEEE Trans MTT., vol. 41, pp. 70-78, 1993.

[6]Pregla, R. and Pascher, W. Numerical Techniques for Microwave and Millimeter Wave Passive Structures. pp. 381-446, John Wiley \& Sons, Inc., New York, (1989).

[7] E.O. Hammerstard, "Equations for Microstrip Circuit Design," in Proceedings of the European Microwave Conference, Hamburg, Germany, pp. 268-272, 1975.

[8] E.O. Hammerstard, and O. Jensen, "Accurate Models for MicrostripComputer-aided design," IEEE MTT-S, Digest, pp.407-409, 1980

[9] H. Wheeler, "Transmission line properties of parallel strips separated by a dielectric sheet," IEEE Trans MTT., vol. 13, 172-185, 1965.

[10] M. Kirsching, and R. H. Jansen, "Accurate Wide-Range Design Equations for the Frequency-Dependent Characteristic of Parallel Coupled Microstrip Lines," IEEE Trans MTT., vol. 23, pp. 83-90, 1984.

[11] D.M. Watson, and K.C. Gupta, "Design and optimization of CPW circuits using EM-ANN models for CPW components," IEEE Trans MTT., vol. 45, 2515-2523, 1997.

[12] F. Devabhaktuni, V.K. Wang and Q.J. Zhang, "A hierarchical neural network approach to the development of a library of neural models for microwave design," IEEE MTT-S, Digest, pp. 1776-1780, 1998.

[13] S. Sagroglu, and C. YIldz, "A multilayered perceptron neural network for a micro-coplanar strip line," Int.Journal of Electromagnetics, 22, 53-563, 2002.

[14] V. Devabhaktuni, M. Yagoub, and Q. J. Zhang, "A robust algorithm for automatic development of neural network models for microwave applications," IEEE Trans MTT., vol.49, pp. 2282-2291, 2001.

[15] Q. J. Zhang and K. C. Gupta. Neural networks for RF and microwave design: Artech House, 2000. 